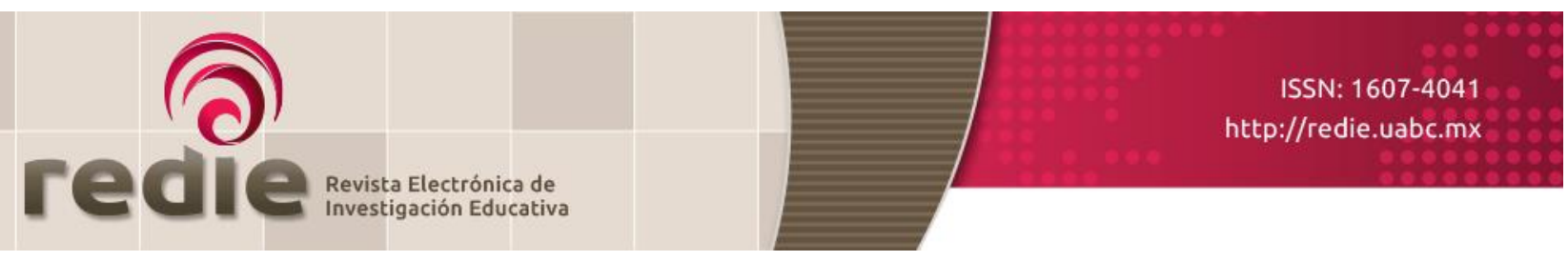

Vol. 21, 2019/e05

\title{
Percepción de estudiantes universitarios sobre el uso de Socrative en experiencias de aprendizaje con tecnología móvil
}

\section{University Students' Perception of the Use of Socrative in Learning Experiences with Mobile Technology}

\author{
Víctor Hugo Perera Rodríguez (*) vhperera@us.es \\ Carlos Hervás Gómez (*) hervas@us.es
}

(*) Universidad de Sevilla

(Recibido: 28 de febrero de 2017; Aceptado para su publicación: 3 de junio de 2017)

Cómo citar: Perera, V. H. y Hervás, C. (2019). Percepción de estudiantes universitarios sobre el uso de Socrative en experiencias de aprendizaje con tecnología móvil. Revista Electrónica de Investigación Educativa, 21, e05, 1-10. doi:10.24320/redie.2019.21.e05.1850

\begin{abstract}
Resumen
La creciente integración de la tecnología móvil en el aula está generando nuevos escenarios para innovar los métodos docentes. En este estudio se analizan las percepciones que maestros en formación muestran específicamente sobre el uso de Socrative y sus implicaciones en procesos de aprendizaje. El diseño de la investigación fue de tipo descriptivo basado en encuestas. En la recogida de datos se administró el cuestionario diseñado ad hoc. Se trabajó sobre una muestra de estudiantes universitarios $(n=168)$ del grado en Educación Infantil de la Facultad de Ciencias de la Educación de una universidad española. Los resultados de este estudio apuntaron cambios significativos en el proceso de aprendizaje de los alumnos, en las relaciones sociales y en la metodología del docente.
\end{abstract}

Palabras clave: Educación Superior, aprendizaje activo, percepción del estudiante, valoración del estudiante.

\begin{abstract}
The growing integration of mobile technology into classrooms is producing new environments that innovate teaching methods. This descriptive, survey-based study analyzes trainee teachers' perceptions of the use of Socrative and its implications for learning processes. Data was collected through a questionnaire designed ad hoc. The study sample comprised university students ( $n=168)$ from the bachelor's degree in early childhood education at the School of Education Sciences of a Spanish university. The results of this study indicate significant changes in students' learning processes, social relations, and teaching methodologies.
\end{abstract}

Key word: Higher Education, activity learning, students' perception, student assessment. 


\section{Introducción}

La integración de la tecnología móvil en las aulas se está dando de forma paulatina, aunque es aún una realidad incipiente en la enseñanza universitaria. En los últimos años, el interés que ha suscitado entre los docentes el uso de aplicaciones basadas en dispositivos móviles para promover el aprendizaje activo e interactivo (Wang, Shen, Novak y Pan, 2009), pero también para conocer y evaluar en tiempo real los aprendizajes de los alumnos, está impulsando numerosas innovaciones en metodología docente. En esta línea, hay estudios que ponen de relieve que las diferentes orientaciones pedagógicas de los docentes afectan a sus métodos de enseñanza, especialmente sus formas de integración de la tecnología en la clase y sus patrones de interacción con los estudiantes (Looi, Sun, Seow, y Chia, 2014).

Las nuevas experiencias educativas auspiciadas por el modelo Bring Your Own Devices (BYOD) -práctica consistente en que los alumnos lleven sus propios dispositivos móviles al lugar de aprendizaje-, no sólo están impulsando el empleo de metodologías creativas (Cochrane, Antonczakb, Keeganc y Narayana, 2014), que resuelven por ejemplo algunos de los problemas que afectan a la gestión del aula (Choudhury, Venkatesh, Bhattacharya, y Sarma, 2016), sino que además están cubriendo muchas de las necesidades que demandan los estudiantes de hoy, conocidos como nativos digitales (Blasco, 2016; Wash, 2014).

Desde la década de los noventa muchos estudios han mostrado interés por el funcionamiento de aplicaciones interactivas basadas en sistemas de respuesta a audiencias en el aula convencional, a estos dispositivos se les conoce como clickers. Actualmente, los sistemas de respuesta inteligente apoyados por servicios en línea se han popularizado y son reconocidos con distintas denominaciones en la literatura científica anglosajona, a saber, entre las principales: audience response system, classroom response system, personal response system o student response system (SRS). Estos sistemas están permitiendo que los docentes formulen preguntas y los estudiantes respondan a las mismas pudiendo obtener un feedback inmediato para el alumnado (Dervan, 2014) e información cualitativa e informes estadísticos para el docente. La evaluación basada en sistemas eficientes de feedback provee a los estudiantes de numerosos beneficios (Debuse y Lawley, 2016) y la utilidad para el docente es indudable puesto que permite conocer in situ y de manera muy rápida el conocimiento que han adquirido los alumnos.

De entre el amplio repertorio de aplicaciones de aprendizaje móvil disponibles actualmente, Socrative es una de las plataformas SRS con mayor aceptación y uso por parte del gremio docente (Haintz, Pichler y Ebner, 2014). Los estudios indican que la utilización de estos sistemas posibilita el aprendizaje activo (Pettit, Mccoy, Kinney y Schwartz, 2015), mejorando la interacción en el aula (Derván, 2014), y confirmándose paralelamente un aumento en la asistencia y participación en clase (Cubric y Jefferies, 2015), en la motivación de los alumnos (Frías, Arce y Flores-Morales, 2016), y en la mejora de resultados académicos en las evaluaciones de los conocimientos de los estudiantes (Trindade, 2014). Además, otros estudios han mostrado que la utilización de este sistema para evaluar no es exclusivo de los docentes, siendo también empleado eficazmente por los estudiantes (Domínguez et al., 2013; Seco y Cardoso, 2015).

Un creciente número de estudios ha abordado el impacto de estos sistemas SRS sobre el rendimiento académico de los estudiantes universitarios (Castillo-Manzano, Castro-Nuño, López-Valpuesta, Sanz-Díaz, e Yñiguez, 2016). Asimismo, se ha estudiado que estas aplicaciones influyen positivamente en las relaciones entre el profesor y los estudiantes, y entre los propios estudiantes, cambiando la naturaleza de las interacciones comunicativas en el aula, optimizando sustancialmente el aprendizaje colaborativo y el compromiso de los estudiantes en clase (Kaya y Balta, 2016; Stowell, 2015) y, en consecuencia, mejorando el rendimiento académico (Awedh, Mueen, Zafar y Manzoor, 2014; Dakka, 2015; Dervan, 2014). En sus estudios, Hunsu, Adesope y Bayly (2016) y Ohashi (2015) encontraron que la utilización de tecnología móvil basada en SRS tuvo efectos escasos aunque significativos en los resultados de aprendizajes cognitivos (memoria, comprensión, resolución de problemas, etc.) y aprendizajes no cognitivos (tiempo de reacción para responder a las preguntas).

Las implicaciones educativas de los sistemas SRS están siendo determinadas por diversos factores. Se han examinado factores de orden tecnológico (en el sentido de usabilidad, portabilidad, disponibilidad de la 
información o acceso a Internet) e individual (por ejemplo, desarrollar, intercambiar y adaptar ideas con otros estudiantes) que afectan positivamente al uso de la tecnología móvil para el aprendizaje (Olufunmilola, Oladimeji y Ayuba, 2016). Otros factores igualmente influyentes han considerado el dominio en el conocimiento de la materia por parte de los estudiantes, el número de asistentes a clases y el empleo frecuente de los dispositivos (Hunsu et al., 2016). En su estudio, Wang (2015) demostró que con el paso del tiempo, y a causa del desgaste, la percepción positiva de los estudiantes sobre los sistemas SRS había cambiado en relación con la facilidad de uso, la concentración, la dinámica participativa de la clase y el aprendizaje percibido, quedando sensiblemente reducidos el compromiso y la motivación.

Los estudios analizados ponen de manifiesto la necesidad de profundizar aún más en las percepciones y valoraciones de estudiantes que han experimentado el uso de estos sistemas SRS en procesos de aprendizaje y de evaluación de conocimientos, con el objetivo de identificar nuevas variables que promuevan los beneficios derivados del uso de estos sistemas en contextos educativos, y tratando de evidenciar los efectos de nuevos factores que reduzcan el potencial metodológico de estas herramientas.

\section{Método}

Esta investigación empleó un diseño no experimental, de tipo descriptivo, basado en encuestas de opinión o survey (Fowler, 2014). El estudio tuvo como finalidad conocer las percepciones que tuvieron estudiantes universitarios utilizando Socrative para el desarrollo de prácticas de aprendizaje en la asignatura "TIC aplicadas a la Educación Infantil". Los objetivos específicos que se plantearon fueron: a) analizar la percepción que tienen los estudiantes respecto a la aplicación Socrative; b) conocer la valoración que emiten los estudiantes sobre el uso de Socrative en situaciones de aprendizaje y de evaluación de conocimientos; y c) determinar los aspectos del uso de Socrative relacionados con la satisfacción.

En el proceso de composición de la muestra se empleó un método de elección no aleatorio; concretamente se llevó a cabo un muestreo no probabilístico causal, en el que el criterio más común de elección de los sujetos que constituyen la muestra se basó en su accesibilidad. Los participantes fueron estudiantes que pertenecían a tres clases de cuarto curso del Grado en Educación Infantil de la Facultad de Ciencias de la Educación de una universidad española. En su generalidad, presentaron características sociodemográficas similares, y sus edades se encontraban por término medio entre los 21 y los 22 años. Inicialmente se contempló que el estudio abarcara los 212 estudiantes matriculados en la asignatura. Sin embargo, sólo se pudieron recabar datos de 168 estudiantes, lo que supuso un tamaño muestral estadísticamente representativo para un nivel de confianza del 95\%, con un error muestral inferior al $+3.5 \%$ y $P=Q$.

\subsection{Procedimiento}

El profesorado adaptó la programación de la asignatura considerando la aplicación Socrative como recurso educativo para apoyar sus métodos de enseñanza y evaluación. La experiencia tuvo lugar durante el curso académico 2016/2017. En este período se llevaron a cabo varias prácticas con directrices y finalidades educativas diferentes.

La tipología de actividades que se programaron para utilizar Socrative en clase abarcó distintas modalidades evaluativas implementadas en un ciclo de tres momentos en torno a una lección o tema de estudio, a saber: a) momento previo a la presentación de un tema, en el que se aplicó una prueba diagnóstica para conocer las ideas que tienen los alumnos sobre los contenidos de un tema determinado; b) momento intermedio, que tuvo lugar en el transcurso de una lección a través de una prueba de competición por grupos sobre el conocimiento de un tema en desarrollo; y c) momento de cierre, en el que se puso en práctica la evaluación del tema tratado a través de una prueba final. En estos tres casos, como puede observarse en la figura 1, los estudiantes respondieron de manera individual o grupal, según las condiciones de la práctica planteada, a una serie de preguntas diseñadas previamente por el profesor en la plataforma Socrative. 


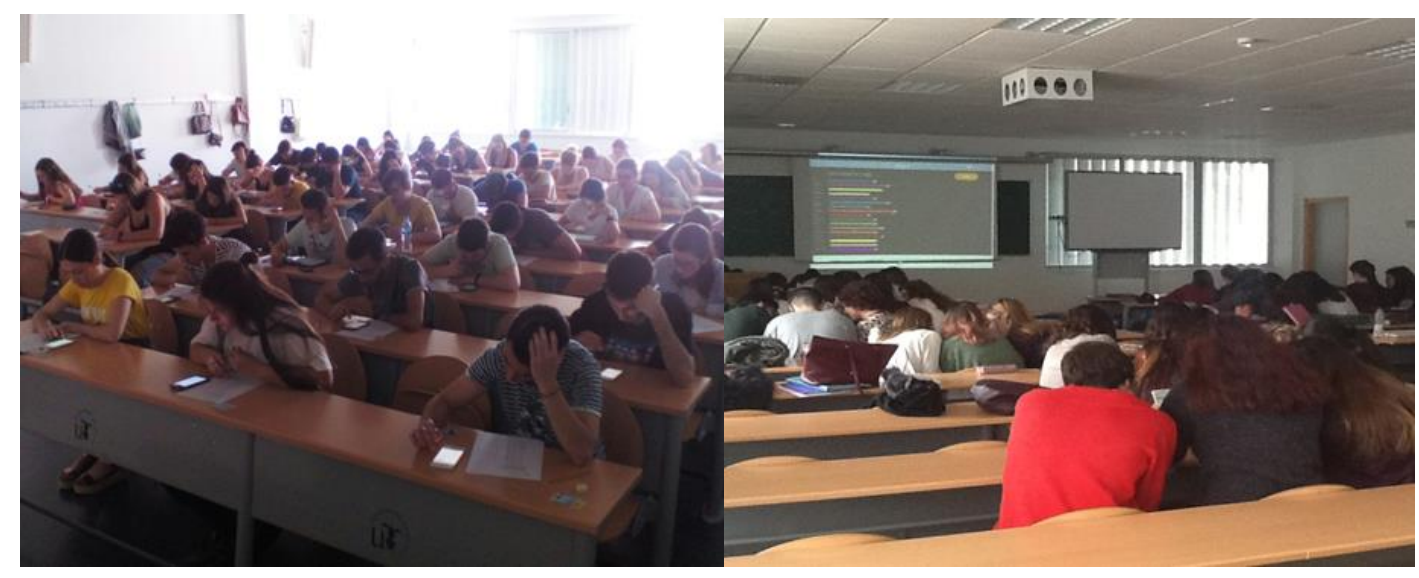

Figura 1. Uso de Socrative en el desarrollo de prácticas individuales y grupales

Las preguntas presentaron un cariz diferente en cuanto a su número, formulación y nivel de complejidad de acuerdo con el tipo de prueba a la que correspondía. De este modo, las encuestas iniciales y finales incluyeron una cantidad dispar de preguntas adaptadas a un tema en cuestión, centrándose en los conceptos teóricos fundamentales que los estudiantes debían dominar; mientras que las pruebas de evaluación por competición animaron a demostrar la teoría que los estudiantes ya debían haber asimilado.

En el primer grupo, las pruebas iniciales enunciaban preguntas abiertas y de opción verdadero/falso; las pruebas finales, por su parte, mostraban preguntas de verificación formuladas con respuestas múltiples. En cuanto a las pruebas de competición, las preguntas fueron siempre de alternativas múltiples.

La plataforma Socrative realizó el registro de las respuestas seleccionadas o escritas por los participantes en tiempo real, ofreciendo al docente, en el primer caso, un informe de los resultados obtenidos que permitió a la postre observar el progreso individual y el análisis comparativo del grupo-aula respecto al nivel de conocimientos en una temática.

\subsection{Instrumento}

La técnica utilizada para la recolección de los datos fue la encuesta. Se emplea la primera edición del cuestionario diseñado recientemente por Quiroga, Fernández, Escorial, Merino y Privado (2016) para valorar la aplicación de Socrative y la experiencia que los estudiantes adquirieron con su uso. El instrumento está compuesto por 19 ítems, medidos en una escala Likert de cinco puntos, y organizados en dos bloques, con los que se pretende, por una parte, conocer la experiencia de aprendizaje con Socrative (ítems 1 al 6) y, por otra, conocer las implicaciones que se derivan de su uso (ítems 7 al 19). El segundo bloque de ítems contempla a su vez tres núcleos teóricos enfocados para medir aspectos pedagógicos (proceso de aprendizaje), sociales (relación docente-alumnos) y metodológicos (tutorización, rendimiento académico, etc.).

Los datos recabados con este instrumento reflejaron en términos generales las percepciones que tuvieron los informantes respecto a cómo experimentaron el uso de Socrative en el contexto de las distintas actividades evaluativas que realizaron en el transcurso de la asignatura. Este instrumento fue elaborado ad hoc por Quiroga et al. (2016). Un análisis de fiabilidad basado en el coeficiente alfa de Cronbach arrojó un valor a de .936, lo que denota un alto grado de consistencia interna de la escala del instrumento. En consecuencia, y en respuesta a los objetivos que se han planteado en este estudio, se mantuvo fielmente la propuesta original de ítems, su estructura y organización. El proceso de administración del instrumento tuvo lugar una semana antes de finalizar el período lectivo, y se consideró oportuno facilitar la recogida de los datos utilizando la tecnología Google Drive, recogiéndose en total las opiniones de 168 estudiantes. 


\subsection{Análisis de los datos}

Concluida la fase de trabajo de campo se procedió al análisis de los datos recogidos. En esta tarea de análisis se trabajó organizando y disponiendo la información del cuestionario en una matriz de datos confeccionada en Excel, de manera que pudiera ser fácilmente exportada al programa spss (versión 22) para su tratamiento estadístico.

Dado que los análisis que habrían de utilizarse tenían que centrarse en los datos recabados a partir del cuestionario referido a la percepción del estudiante sobre experiencia de aprendizaje en las prácticas desarrolladas con Socrative, se procedió al análisis estratégico del cálculo de la frecuencia en las respuestas a cada uno de los ítems, considerando en su presentación los dos bloques diferenciados a los que hacía referencia el cuestionario, así como las tres categorías que constituían el segundo bloque de ítems.

De acuerdo con el proceso descrito para el tratamiento de los datos, y en respuesta a uno de los objetivos propuestos, se analizó igualmente el sentido y el grado en el que se relacionaron algunas variables de interés, realizando para ello un análisis correlacional.

\section{Resultados}

De acuerdo con los objetivos enunciados en la investigación, se presentan los resultados estadísticos más relevantes atendiendo al conjunto de ítems del cuestionario.

En el primer objetivo se planteó analizar las percepciones que tienen los estudiantes respecto a la aplicación Socrative. De acuerdo con los resultados que se presentan en la tabla I, puede afirmarse que los encuestados perciben que esta aplicación es fácil de utilizar (ítem $5, \bar{x}=4.21$ ), aunque se aprecia cierta dificultad en el uso de esta herramienta cuando se accede a la aplicación con el perfil de profesor (ítem 2, $\bar{x}=3.54$ ) frente al modo de acceso con el perfil de alumno (ítem $1, \bar{x}=4.04$ ). Es probable que esta importante diferencia se deba a que el grueso de sus experiencias en Socrative se han desarrollado como alumno, gracias a que se realizaron distintas prácticas en la asignatura. En ninguna actividad se requería que accedieran como profesor y, por tanto, que algunos conozcan la aplicación bajo este perfil se debe a su iniciativa e interés personal. Un segundo aspecto que define a esta aplicación es, desde la perspectiva de sus usuarios, su potencial capacidad motivadora (ítem $6, \bar{x}=3.80, \delta=1.03$ ). Aunque no todos los alumnos están convencidos de que esta aplicación por sí misma resulte motivadora, por lo que dicha capacidad queda a juicio del tipo de práctica que se realice.

Tabla I. Opiniones de los estudiantes universitarios sobre el uso de SRS

\begin{tabular}{|c|c|c|}
\hline ítems del cuestionario & $\overline{\boldsymbol{x}}$ & $\delta$ \\
\hline 1. Me ha resultado fácil utilizar la sRs de alumnos. & 4.04 & .89 \\
\hline 2. He podido utilizar la sRS de profesores sin mayores dificultades. & 3.54 & 1.04 \\
\hline 3. Creo que sRs me ha ayudado a reflexionar sobre los conceptos impartidos sobre el tema tratado. & 3.57 & .90 \\
\hline 4. En general, puedo afirmar que este primer contacto con SRS me ha resultado satisfactorio. & 3.93 & .98 \\
\hline 5. En general, creo que sRs es fácil de utilizar. & 4.21 & .88 \\
\hline 6. En general, la utilización de sRs es motivadora en sí misma. & 3.80 & 1.03 \\
\hline
\end{tabular}

Nota: $n=168$, número de estudiantes que responden a la escala Likert (1-5 puntos) en respuesta a la primera categoría.

El segundo objetivo pretendía conocer la valoración que emiten los estudiantes sobre el uso de Socrative en situaciones de aprendizaje y de evaluación de sus conocimientos. En este sentido, los resultados de la tabla II toman en consideración las tres áreas teóricas que mejor explican esta dimensión, más amplia de su experiencia de uso. En primer lugar, cabe destacar el formidable beneficio que ofrece la herramienta para que los alumnos promuevan determinadas actividades cognitivas asociadas a su proceso de aprendizaje, como son sentirse más motivados (ítem $8, \bar{x}=3.84$ ) y prestar más atención en clase (ítem 7 , $\bar{x}=3.79$ ), asimilar los contenidos de un tema (ítem 10, $\bar{x}=3.75$ ), recordar sus conceptos básicos (ítem 11 , 
$\bar{x}=3.68$ ) y reflexionar sobre estos (ítem $3, \bar{x}=3.57$ ), lo que se traduce finalmente en la posibilidad de que los alumnos puedan demostrar un buen rendimiento académico (ítem $15, \bar{x}=3.70$ ), y, tenga en su haber una herramienta útil para el proceso de aprendizaje (ítem 19, $\bar{x}=4.18$ ). En segundo lugar, Socrative fue percibido por los estudiantes como un recurso que aumenta las interacciones académicas en el aula, haciendo que mejore en este sentido la relación con el docente (ítem 17, $\bar{x}=3.77$ ), gracias en parte al feedback que pudiera requerir el tratamiento de las respuestas (ítem 9, $\bar{x}=3.59$ ); y la relación entre los propios alumnos (ítem 13, $\bar{x}=3.71$ ). Por último, utilizado como método de aprendizaje, Socrative tiene la capacidad de transformar la imagen del docente acercándolo a un perfil competencial en tecnología más adaptado a la sociedad digital en la que se desenvuelven los estudiantes (ítem 12, $\bar{x}=4.16$ ), y también abrir a escenarios menos convencionales, más digitalizados, el propio contexto formal en el que aprenden (ítem $14, \bar{x}=4.14)$. En opinión de los alumnos, no sólo hace que el aprendizaje sea más activo (ítem 16, $\bar{x}=4.04$ ), sino que posibilita a su vez que puedan tener un mayor control de su aprendizaje, en aras de una mayor autonomía (ítem $18, \bar{x}=3.82$ ).

Tabla II. Opiniones de los estudiantes universitario sobre las implicaciones del uso de SRS

\begin{tabular}{|c|c|c|}
\hline Ítems del cuestionario & $\bar{x}$ & $\delta$ \\
\hline 7. Creo que la aplicación de SRS podría ayudar a los alumnos a prestar más atención en las clases. & 3.79 & .86 \\
\hline 8. Pienso que los alumnos estarían más motivados en las clases en las que se utilizara SRS. & 3.84 & .90 \\
\hline 9. SRS posibilitaría una tutela inmediata de los alumnos (feedback). & 3.59 & .65 \\
\hline $\begin{array}{l}\text { 10. Estimo que SRS podría ayudar a asimilar mejor los contenidos impartidos en las diferentes } \\
\text { asignaturas. }\end{array}$ & 3.75 & .91 \\
\hline 11. Juzgo que con SRS es posible recordar mejor los conceptos desarrollados por el profesor. & 3.68 & .85 \\
\hline 12. Si los profesores utilizaran SRS, éstos aparecerían más cercanos al actual mundo digitalizado. & 4.16 & .86 \\
\hline 13. SRS posibilitaría una mayor interacción académica entre los propios alumnos. & 3.71 & .90 \\
\hline $\begin{array}{l}\text { 14. Creo que la utilización de SRS acerca más el contexto formal de enseñanza-aprendizaje al mundo } \\
\text { digitalizado. }\end{array}$ & 4.14 & .86 \\
\hline 15. Infiero que SRS podría contribuir a mejorar el rendimiento académico de los alumnos. & 3.70 & .73 \\
\hline 16. SRS abriría posibilidades nuevas para un aprendizaje más activo por parte de los alumnos. & 4.04 & .73 \\
\hline 17. Con SRS mejoraría la interacción profesor/alumno. & 3.77 & .85 \\
\hline $\begin{array}{l}\text { 18. Considero que SRS posibilitaría un mayor control por parte de cada alumno en su proceso de } \\
\text { aprendizaje. }\end{array}$ & 3.82 & .83 \\
\hline 19. En general, creo que SRS puede resultar útil para el proceso de aprendizaje. & 4.18 & .83 \\
\hline
\end{tabular}

Nota: $n=168$, número de estudiantes que responden a la escala Likert (1-5 puntos) en respuesta a la segunda y tercera categorías.

En relación con el tercer objetivo formulado, se pretendió determinar los aspectos del uso de Socrative que guardaban relación significativa con la percepción de satisfacción que manifiestaban los alumnos (ver tablas III y IV). Tras aplicar la prueba para la obtención de coeficiente de correlación de Pearson, puede afirmarse, con un nivel de confianza del 99\% ( $p<0.01)$, que existe una fuerte correlación entre el grado alto de satisfacción y las variables: fácil de utilizar ( $r=.722)$, aplicación motivadora en sí misma ( $r=.751)$, profesores con competencia tecnológica $(r=.712)$, apertura del aprendizaje formal $(r=.717)$ y utilidad de la aplicación ( $(=.744)$. 
Tabla III. Correlación entre "uso de Socrative" y "percepción de satisfacción".

\begin{tabular}{|c|c|c|c|c|c|c|c|c|c|}
\hline & Ítem 1 & Ítem 2 & Ítem 3 & Ítem 4 & Ítem 5 & Ítem 6 & Ítem 7 & Ítem 8 & Ítem 9 \\
\hline Ítem 2 & $.252^{\pi x}$ & & & & & & & & \\
\hline Ítem 3 & $.376^{\star \star}$ & $.284^{* *}$ & & & & & & & \\
\hline Ítem 4 & $.518^{* *}$ & $.355^{* *}$ & $.591^{* *}$ & & & & & & \\
\hline Ítem 5 & $.540^{* *}$ & $.442^{* *}$ & $.519^{* *}$ & $.722^{* *}$ & & & & & \\
\hline Ítem 6 & $.401^{* \star}$ & $.335^{* *}$ & $.584^{* *}$ & $.751^{* *}$ & $.680^{* *}$ & & & & \\
\hline Ítem 7 & $.409^{\star *}$ & $.270^{* *}$ & $.571^{* \star}$ & $.533^{* *}$ & $.485^{* *}$ & $.661^{* *}$ & & & \\
\hline Ítem 8 & $.343^{\star \star}$ & $.303^{* *}$ & $.485^{* *}$ & $.674^{* *}$ & $.605^{\star *}$ & $.700^{* *}$ & $.738^{\star *}$ & & \\
\hline Ítem 9 & $.212^{* *}$ & $.195^{*}$ & $.370^{* *}$ & $.207^{* *}$ & $.185^{*}$ & $.362^{* *}$ & $.418^{* *}$ & $.376^{\star *}$ & \\
\hline Ítem 10 & $.343^{* *}$ & $.275^{* *}$ & $.586^{* *}$ & $.580^{* *}$ & $.490^{* *}$ & $.674^{* *}$ & $.615^{* *}$ & $.646^{* *}$ & $.431^{* * x}$ \\
\hline Ítem 11 & $.467^{* *}$ & $.258^{* *}$ & $.730^{* *}$ & $.618^{* *}$ & $.547^{* *}$ & $.565^{* *}$ & $.665^{* *}$ & $.563^{* *}$ & $.377^{* *}$ \\
\hline Ítem 12 & $.414^{\star *}$ & $.485^{* *}$ & $.662^{* *}$ & $.712^{\star *}$ & $.661^{* *}$ & $.703^{* *}$ & $.577^{* *}$ & $.585^{* \star}$ & $.310^{* *}$ \\
\hline Ítem 13 & $.393^{* \star}$ & $.203^{* *}$ & $.376^{* *}$ & $.463^{* \star}$ & $.302^{* *}$ & $.500^{* *}$ & $.359^{* *}$ & $.317^{\star \star}$ & $.227^{* *}$ \\
\hline Ítem 14 & $.442^{* *}$ & $.560^{* *}$ & $.612^{* *}$ & $.717^{\star *}$ & $.624^{* *}$ & $.664^{* *}$ & $.528^{* *}$ & $.632^{* *}$ & $.299^{* *}$ \\
\hline Ítem 15 & $.321^{* *}$ & $.286^{* *}$ & $.615^{* *}$ & $.444^{* *}$ & $.518^{* *}$ & $.541^{* *}$ & $.607^{* *}$ & $.523^{* *}$ & $.529^{* *}$ \\
\hline Ítem 16 & $.467^{\star \star}$ & $.376^{* *}$ & $.591^{\star *}$ & $.552^{\star *}$ & $.598^{* *}$ & $.581^{\star *}$ & $.665^{* x}$ & $.659^{\star \star}$ & $.408^{* \pi}$ \\
\hline Ítem 17 & $.178^{*}$ & . 142 & $.384^{* *}$ & $.282^{\star *}$ & $.283^{\star *}$ & $.380^{* *}$ & $.325^{* \star}$ & $.232^{\star \star}$ & $.413^{* *}$ \\
\hline Ítem 18 & $.521^{\star *}$ & .133 & $.424^{* *}$ & $.448^{* *}$ & $.298^{* *}$ & $.380^{* *}$ & $.323^{* *}$ & $.225^{* *}$ & $.363^{* *}$ \\
\hline Ítem 19 & $.479^{* *}$ & $.390^{* *}$ & $.653^{* *}$ & $.744^{* *}$ & $.708^{* *}$ & $.716^{* *}$ & $.657^{* *}$ & $.614^{* *}$ & $.337^{* *}$ \\
\hline
\end{tabular}

Nota: $\mathrm{n}=168,{ }^{*} p<.05,{ }^{* *} p<.01$. Ver Tabla I y Tabla II

Tabla IV. Correlación entre "uso de Socrative" y "percepción de satisfacción" (continuación)

\begin{tabular}{|c|c|c|c|c|c|c|c|c|c|}
\hline & Ítem 10 & Ítem 11 & Ítem 12 & Ítem 13 & Ítem 14 & Ítem 15 & Ítem 16 & Ítem 17 & Ítem 18 \\
\hline Ítem 11 & $.682^{\text {x* }}$ & & & & & & & & \\
\hline Ítem 12 & $.643^{* *}$ & $.682^{* *}$ & & & & & & & \\
\hline ítem 13 & $.435^{* *}$ & $.465^{* *}$ & $.404^{* *}$ & & & & & & \\
\hline Ítem 14 & $.619^{* *}$ & $.557^{* *}$ & $.818^{* *}$ & $.308^{* *}$ & & & & & \\
\hline ítem 15 & $.718^{* *}$ & $.708^{\star *}$ & $.646^{* *}$ & $.384^{* *}$ & $.556^{\star *}$ & & & & \\
\hline ítem 16 & $.657^{* *}$ & $.682^{\star *}$ & $.671^{* *}$ & $.422^{* *}$ & $.564^{* *}$ & $.723^{* *}$ & & & \\
\hline Ítem 17 & $.458^{* *}$ & $.469^{* *}$ & $.395^{* *}$ & $.664^{* *}$ & $.170^{*}$ & $.465^{* *}$ & $.447^{* *}$ & & \\
\hline Ítem 18 & $.463^{* *}$ & $.454^{* *}$ & $.316^{* *}$ & $.603^{* *}$ & $.315^{* *}$ & $.473^{* *}$ & $.395^{* *}$ & $.529^{* *}$ & \\
\hline ítem 19 & $.795^{* *}$ & $.796^{* *}$ & $.763^{* *}$ & $.525^{* *}$ & $.723^{* *}$ & $.682^{* *}$ & $.670^{* *}$ & $.520^{* *}$ & $.492^{* *}$ \\
\hline
\end{tabular}

Nota: $\mathrm{n}=168,{ }^{*} p<.05, * * p<.01$.

\section{Discusión y conclusiones}

Las conclusiones más relevantes de este estudio están en consonancia con los resultados definitivos de los trabajos de Quiroga et al. (2016), quienes sugieren la implantación del SRS como sistema de apoyo al aprendizaje y a la evaluación de conocimientos en la metodología docente por sus cuantiosos beneficios e implicaciones pedagógicas. En líneas generales, la percepción del alumnado sobre la aplicación Socrative es positiva en todos los aspectos evaluados (por ejemplo, pedagógico, social y metodológico), destacándose, en última instancia, su valiosa utilidad en la actividad de aprendizaje.

Cabe destacar los efectos directos que tiene el uso de Socrative para incentivar el aprendizaje social e implicar al docente en una relación más próxima, fluida y académicamente significativa para el alumno a través de su tutela inmediata (Awedh et al., 2014; Dakka, 2015; Dervan, 2014; Frías et al., 2016; Trindade, 2014). Además, el empleo de los dispositivos móviles en el aula, que dan sustento y funcionalidad a esta aplicación, hace posible conectar convenientemente el mundo digital en el que vive el alumno con el contexto formal universitario en el que aprende, habitualmente considerado un espacio reticente a desarrollar prácticas educativas con este tipo de tecnologías. 
Las nuevas experiencias educativas que están surgiendo gracias al empleo de los dispositivos móviles no sólo están mitigando los problemas que afectan a la gestión del aula, como son la masificación de estudiantes, la baja tasa de participación y la desmotivación (Blasco-Arcas, Buil, Hernández-Ortega y Sese, 2013), sino que además parecen estar cubriendo las necesidades que vienen demandando los estudiantes de hoy en términos de aprendizaje (Blasco, 2016; Wash y Freeman, 2013).

De acuerdo con los resultados de algunos estudios previos (González-Fernández y Salcines-Talledo, 2015; Hadiri, 2015; Hanus y Fox, 2015), cabe mencionar el importante papel que juega este tipo de aplicaciones ofreciendo a los alumnos nuevos escenarios formativos que dan oportunidades para que puedan sentirse más motivados y satisfechos; además de ser más participativos, autónomos y conscientes de su capacidad para poder supervisar y autorregular su propio aprendizaje. En definitiva, la aplicación Socrative, tal como se ha demostrado en otros estudios (Kim, Rueckert, Kim y Seo, 2013), parece proporcionar una experiencia de aprendizaje más personalizada, donde los estudiantes pueden involucrarse activamente en el control de su aprendizaje por medio de un conjunto programado de preguntas que pueden provocar retos cognitivos.

Se sugiere que en futuras investigaciones se profundice en los factores críticos de éxito en el uso pedagógico de Socrative con dispositivos móviles de tal forma que faciliten las actividades cognitivas involucradas en el proceso de aprendizaje de los estudiantes.

\section{Alcance y limitaciones del estudio}

Las conclusiones de este estudio refuerzan la relación entre la tecnología móvil y el aprendizaje en el aula universitaria. El empleo de los dispositivos móviles en el aula no sólo está dando cobertura al modelo BYOD, también está proporcionando al docente la capacidad de generar nuevos escenarios en beneficio del proceso de aprendizaje. En este sentido, están emergiendo espacios de interés relacionados con el diseño de actividades apoyadas en dispositivos móviles que traerán consigo nuevos retos y futuras líneas de investigación.

En la práctica pedagógica, Socrative está siendo uno de los recursos tecnológicos empleados para apoyar la formación en las aulas universitarias con consecuencias significativas en el ámbito metodológico y de la evaluación. Así, su utilización no solo está mejorando la participación y comunicación entre los agentes educativos, promoviendo a su vez prácticas activas que facilitan los procesos de aprendizaje, sino que está concibiendo la evaluación como un proceso permanente de monitoreo de los aprendizajes, lo que implica que se pueda atender mejor las necesidades educativas de los alumnos.

Por último, los autores reconocen las limitaciones de este estudio en relación al abordaje que se hace de la realidad educativa desde el enfoque cuantitativo. La investigación necesitaría de complementariedad metodológica, incorporando técnicas cualitativas en la recogida de datos que ayudaran a profundizar en los distintos aspectos analizados desde las voces de los propios estudiantes.

\section{Referencias}

Awedh, M., Mueen, A., Zafar, B. y Manzoor, U. (2014). Using Socrative and smartphones for the support of collaborative learning. International Journal on Integrating Technology in Education, 3(4), 17-24.

doi:10.5121/ijite.2014.3402

Blasco, D. (2016). Student's attitudes toward integrating mobile technology into translation activities. International Journal on Integrating Technology in Education, 5(1), 1-11. doi:10.5121/ijite.2016.5101

Blasco-Arcas, L., Buil, I., Hernández-Ortega, B. y Sese, F. J. (2013). Using clickers in class. The role of interactivity, active collaborative learning and engagement in learning performance. Computers \& Education, 62, 102-110. doi:10.1016/j.compedu.2012.10.019 
Castillo-Manzano, J. I., Castro-Nuño, M., López-Valpuesta, L., Sanz-Diáz, M. T. e Yñiguez, R. (2016). Measuring the effect of ARS on academic performance: a global meta-analysis. Computers \& Education, 96(C), 109-121. doi:10.1016/j.compedu.2016.02.007

Choudhury, N., Venkatesh, T., Bhattacharya, S. y Sarma, S. (2016). Avabodhaka: a system to analyse and facilitate interactive learning in an ICT based system for large classroom. Procedia Computer Science, 84, 160-168. doi:10.1016/j.procs.2016.04.082

Cochrane, T. D., Antonczakb, L., Keeganc., H. y Narayana, V. (2014). Riding the wave of Byod: developing a framework for creative pedagogies. Research in Learning Technology, 22. doi: 10.3402/rlt.v22.24637

Cubric, M. y Jefferies, A. (2015). The benefits and challenges of large-scale deployment of electronic voting systems: University student views from across different subject groups. Computers \& Education, 87(C), 98-111. doi:10.1016/j.compedu.2015.04.004

Dakka, S. M. (2015). Using Socrative to enhance in-class student engagement and collaboration. International Journal on Integrating Technology in Education, 4(3), 13-19. doi:10.5121/ijite.2015.4302

Debuse, J. y Lawley, M. (2016). Benefits and drawbacks of computer-based assessment and feedback systems: student and educator perspectives. British Journal of Educational Technology, 47(2), 294-301. doi:10.1111/bjet.12232

Dervan, P. (2014). Enhancing In-class student engagement using Socrative (an online student response system): a report. The All Ireland Journal of Teaching \& Learning in Higher Education, 6(3), 1801-1813.

Domínguez, A., Saenz-de-Navarrete, J., de Marcos, L., Fernández-Sanz, L., Pagés, C. y Martínez-Herráiz, J. J. (2013). Gamifying learning experiences: practical implications and outcomes. Computers \& Education, 63, 380-392. doi:10.1016/j.compedu.2012.12.020

Fowler, F. J. (2014). Survey research methods (5th ed.). Los Ángeles, CA: Sage.

Frías, M. V., Arce, C. y Flores-Morales, P. (2016). Uso de la plataforma socrative.com para alumnos de Química General. Educación Química, 27(1), 59-66. doi:10.1016/j.eq.2015.09.003

González-Fernández, N. y Salcines-Talledo, I. (2015). El smatphone en los procesos de enseñanzaaprendizaje-evaluación en Educación Superior. Percepciones de docentes y estudiantes. Relieve, 21(2). doi:10.7203/relieve.21.2.7480

Hadiri, Y. (2015, enero). Instructional design project: click it to check it. Socrative for student assessment in higher education. Documento presentado en The Technology, Colleges, and Community Worldwide Online Conference. Recuperado de http://hdl.handle.net/10125/35948

Haintz, C., Pichler, K. y Ebner, M. (2014). Developing a web-based question-driven audience response system supporting BYOD. Journal of Universal Computer Science, 20(1), 39-56. doi:10.3217/jucs-020-010039

Hanus, M. D. y Fox, J. (2015). Assesing the effects of gamification in the classroom: a longitudinal study on intrinsic motivation, social comparison, satisfaction, effort, and academic performance. Computer $y$ Education, 80(C), 152-161. doi:10.1016/j.compedu.2014.08.019

Hunsu, N. J., Adesope, O. y Bayly, D. J. (2016). A meta-analysis of the effects of audience response systems (clicker-based technologies) on cognition and affect. Computers \& Education, 94, 102-119.

doi:10.1016/j.compedu.2015.11.013 
Kaya, A. y Balta, N. (2016). Taking advantages of technologies: using the Socrative in english language teaching classes. International Journal of Social Sciences y Educational Studies, 2(3), 4-12.

Kim, D., Rueckert, D., Kim, D.-J., y Seo, D. (2013). Students' perceptions and experiences of mobile learning. Language Learning y Technology, 17(3), 52-73. Recuperado de http://www.lltjournal.org/item/2825

Looi, C.-K., Sun, D., Seow, P. y Chia, G. (2014). Enacting a technology-based science curriculum across a grade level: the journey of teachers' appropriation. Computers \& Education, 71, 222-236.

doi:10.1016/j.compedu.2013.10.006

Ohashi, L. (2015). Enhancing EFL writing courses with the online student response system Socrative. Kokusaikeiei Bunkakenkyu, Cross-cultural Business and Cultural Studies, 19(1), 135-145.

Olufunmilola, O., Oladimeji, F. y Ayuba, D. (2016). Individual and technological factors affecting undergraduates' use of mobile technology in University of Ilorin, Nigeria. Digital Education Review, 29, 124-133. Recuperado de http://revistes.ub.edu/index.php/der/article/view/12039/pdf

Pettit, R. K., Mccoy, L., Kinney, M. y Schwartz, F. N. (2015). Student perceptions of gamified audience response system interactions in large group lectures and via lecture capture technology. BMC Medical Education, 15(1), 1-15. doi:10.1186/s12909-015-0373-7

Quiroga, M. A., Fernández, J., Escorial, S., Merino, M. D. y Privado, J. (2016). Uso de móviles y tabletas para la evaluación de los conocimientos adquiridos: hagamos asequible la evaluación continua (2a. Fase) (Informe del proyecto de Innovación Docente No. 30). Madrid: UCM. Recuperado de https://eprints.ucm.es/34893/

Seco, C. y Cardoso, T. (2015). Questionários sistemáticos e smartphone: ferramentas de avaliação pedagógica? [Cuestionarios sistemáticos y smartphone: ¿herramientas de evaluación pedagógica?]. Revista de estudios e investigación en Psicología y Educación, Extr.(13), 158-162.

doi:10.17979/reipe.2015.0.13.489

Stowell, J. (2015). Use of clickers vs. mobile devices for classroom polling. Computers \& Education, 82(C), 329-334. doi:10.1016/j.compedu.2014.12.008

Trindade, J. (2014). Promoção da interatividade na sala de aula com Socrative: estudo de caso [Promoción de la interactividad en el aula con Socrative: estudio de caso]. Indagatio Didactica, 6(1), 254-268.

Wang, A. I. (2015). The wear out effect of a game-based student response system. Computers \& Education, 82(C), 217-227. doi:10.1016/j.compedu.2014.11.004

Wang, M., Shen, R., Novak, D. y Pan, X. (2009). The impact of mobile learning on students' learning behaviours and performance: report from a large blended classroom. British Journal of Educational Technology, 40(4), 673-695. doi: 10.1111/j.1467-8535.2008.00846.x

Wash, P. D. (2014). Taking advantage of mobile devices: using Socrative in the classroom. Journal of Teaching and Learning with Technology, 3(1), 99-101. doi:10.14434.jotlt.v3n1.5016

Wash, P. D. y Freeman, G. G. (2013). BYOD-Engaging students using their own devices. National Social Science Technology Journal, 3(1). Recuperado de https://www.nssa.us/tech journal/volume 3-1/vol3-1 article8.htm 